\title{
ASYMPTOTIC SOLUTIONS OF CERTAIN LINEAR DIFFERENTIAL EQUATIONS IN WHICH THE COEFFICIENT OF THE PARAMETER MAY HAVE A ZERO*
}

\author{
BY \\ HENRY SCHEFFE
}

Chapter I. The given equation

1. Introduction. Equations of the type

$$
\frac{d^{n} u}{d z^{n}}+p_{1}(z) \frac{d^{n-1} u}{d z^{n-1}}+p_{2}(z) \frac{d^{n-2} u}{d z^{n-2}}+\cdots+\left\{p_{n}(z)-\rho^{n} \phi^{n}(z)\right\} u=0
$$

have been studied by various mathematicians, including Birkhoff, $\uparrow$ Schlesinger, and Horn, with regard to the asymptotic forms of the solutions for large complex values of the parameter $\rho$. The second-order case goes back to Liouville. The essential differences between the present treatment and previous treatments of the $n$ th-order case are the following:

(1) Before this it has been necessary to assume the coefficient $\phi^{n}(z)$ to be bounded from zero. We permit it to have a zero of any integral order $\nu$.

(2) The variable $z$ has been confined to a finite interval of the real axis. We allow it to vary over complex values in a finite region.

(3) In order that the forms be valid for all $\rho$ in the neighborhood of $\rho=\infty$, it has been necessary to require that the coefficient $\phi^{n}(z)$ be real, except possibly for a constant imaginary factor. We dispense with this restriction.

Besides proving the existence of solutions asymptotic to $n$ simple independent expressions, we consider the following problem arising from the first of the above generalizations: What is the form of a solution determined by boundary values at the point where the coefficient $\phi^{n}(z)$ has its zero? At a point where $\phi^{n}(z)$ is different from zero the problem becomes elementary, since it is always possible to find a non-singular matrix of simple forms for $n$ solutions and their first $n-1$ derivatives which is valid in some neighborhood. including the point. But in our case the simple forms are not valid at the zero. The problem is complicated by the Stokes phenomenon, that is, the discontinuity, as a solution is taken across a "Stokes line," of the coefficients

\footnotetext{
* Presented to the Society, April 20, 1935; received by the editors July 3, 1935.

$\dagger$ Birkhoff, On the asymptotic character, etc., these Transactions, vol. 9 (1908), p. 219, which contains references to the work of the other mathematicians mentioned above.
} 
(hereafter called "Stokes multipliers") that multiply the simple forms in the asymptotic expression of the solution.

The case for $n=2$ has been studied by Langer.* Applications of his work not only have led to new results for some of the classical equations of physics (Bessel, ${ }^{*}$ Weber, $\dagger$ Hermite, $\dagger$ Mathieu $\ddagger$ ) but are of importance in quantum physics. $\$$ Mathematically, perhaps the most alluring aspect of the problem is the Stokes phenomenon. In going to the $n$ th-order case it is then of interest to discover how the quantitative dependence of the Stokes multipliers on $\nu$ and how the location of the Stokes lines generalize.

Langer was able to obtain a "related equation" solvable by Bessel functions and thus utilize the classical knowledge of their asymptotic properties. Because of the apparent lack of a suitable standard equation of the $n$th order for which the Stokes multipliers for the principal solutions are known, a considerable part of the work lies in developing this necessary adjunct. A feature of interest is the way in which the multipliers are found, by use of the Stokes formula. In his letter\| in the Abel memorial edition of the Acta Mathematica, Stokes forecasts the general applicability of this method of finding the multipliers for solutions appropriately expressible as power series with gamma function coefficients. The labor of calculating formal solutions at $\infty$ would be lessened in such cases, since the formula yields the leading terms, and the existence of true solutions asymptotic to the formal solutions could then be predicated upon one of the fundamental papers in that field. 1

2. Canonical form. We assume the equation to be treated has undergone preliminary transformations leaving it in normal form and transferring the zero of $\phi^{n}(z)$ to the origin. The given equation

* Langer, On the asymptotic solutions, etc., these Transactions, vol. 33 (1931), p. 23, and vol. 34 (1932), p. 447. In the former article, but not the latter, $z$ is restricted to real values. The region of validity $R_{z}$ need not be finite and $\nu$ need only be positive, where $\phi^{n}(z) \equiv\left(z-z_{0}\right)^{\nu} \phi_{1}{ }^{n}(z)$ and $\phi_{1}{ }^{n}(z)$ is analytic and bounded from zero in $R_{\mathbf{z}}$. See also his symposium article, The asymptotic solutions, etc., Bulletin of the American Mathematical Society, vol. 40 (1934), p. 545, which contains further references to his work.

$\dagger$ N. Schwid, The asymptotic forms of the Hermite and Weber functions, these Transactions, vol. 37 (1935), p. 339.

$\ddagger$ Langer, The solutions of the Mathieu equation, etc., these Transactions, vol. 36 (1934), p. 637.

$\S$ Birkhoff, Quantum mechanics and asymptotic series, Bulletin of the American Mathematical Society, vol. 39 (1933), p. 681.

|| Stokes, On the discontinuity of arbitrary constants, etc., Acta Mathematica, vol. 26 (1902), p. 393.

T Trjitzinsky, Analytic theory of linear differential equations, Acta Mathematica, vol. 62 (1933), p. 167, treats the subject with the greatest generality. The pioneer article is by Poincaré, Sur les intégrales irrégulières des équations linéaires, Acta Mathematica, vol. 8 (1886), p. 295. Important advances were made by Horn, Birkhoff. 


$$
u^{(n)}(z, \rho)+0+p_{2}(z) u^{(n-2)}(z, \rho)+\cdots+\left\{p_{n}(z)-\rho^{n} \phi^{n}(z)\right\} u(z, \rho)=0
$$

is then subject to five hypotheses:

(i) $R_{z}$ is a finite, closed, simply connected region, containing $z=0$ as interior point.

(ii) The coefficients $\phi^{n}(z)$ and $p_{j}(z), j=2,3, \cdots, n$, are analytic in $R_{z}$.

(iii) The coefficient $\phi^{n}(z)$ is of the form $z^{v} \phi_{1}^{n}(z)$, where $\nu$ is a positive integer or zero, and $\phi_{1}^{n}(z) \neq 0$ in $R_{z}$.

Hypothesis (iv), given in $\S 5$, implies a further restriction on the coefficient $\phi^{n}(z)$, while $(v)$, which is most conveniently stated in $\S 8$, further qualifies the region $R_{z}$.

\section{Chapter II. An important Special CaSe}

3. The $t$-equation: two sets of solutions. The simplest possible equation of the type (1) is

$$
\frac{d^{n}}{d x^{n}} y(x, \rho)-\rho^{n} x^{\nu} y(x, \rho)=0,
$$

and we shall consider this first. However, we shall find that the treatment of the general case can be based directly on the results for this special case.

It is preferable to study the simpler transform

$$
\frac{d^{n}}{d t^{n}} y(t)-t^{\nu} y(t)=0
$$

which we shall designate the t-equation, and which we shall later force to serve as our standard equation of the $n$th order. The transformation to the $t$-equation is achieved by the substitution

where

$$
x=\rho^{-n / p} t,
$$

$$
p \equiv n+\nu \text {. }
$$

Power series for that set of solutions of (2) which is principal at the origin were obtained by an elegant method by M. H. Molins* in 1876; they are also easily found by the usual method. The series $\dagger$

* Mémoires de l'Académie des Sciences, Inscriptions et Belles-Lettres de Toulouse, (7), vol. 8 (1876), p. 167.

$\dagger$ If this formula becomes indeterminate at $t=0$ we define $y_{j}{ }^{(m)}(0) \equiv \lim _{t \rightarrow 0} y_{j}{ }^{(m)}(t)$. We use the notation

$$
(a)_{m} \equiv a(a-1)(a-2) \cdots(a-m+1) \quad(m=1,2,3, \cdots) ;(a)_{0} \equiv 1 .
$$

Note $(j)_{m}=0$ if $j$ is an integer $<m$. 


$$
y_{j}{ }^{(m)}(t) \equiv \frac{t^{j-m}}{i !}\left[(j)_{m}+\sum_{l=1}^{\infty} \frac{(l p+j)_{m}}{\prod_{k=1}^{l}(k p+j)_{n}} t^{l p}\right] \quad(j, m=0,1, \cdots, n-1)
$$

for the principal solutions and their derivatives converge for all $t$.

For later use it is desirable to express the coefficients of these power series as quotients of gamma functions. It is also of subsequent advantage to study the solutions of the $t$-equation on a $\xi$-plane by means of the mapping

$$
t \equiv\left(\frac{p}{n} \xi\right)^{n / p}
$$

In the $\xi$-plane it is necessary to construct a Riemann surface with branch point at the origin, appropriate to the single-valued representation of $\arg \xi$. It will suffice to employ the sector

$$
-Q \frac{\pi}{n} \leqq \arg \xi \leqq Q \frac{\pi}{n} .
$$

$Q$ is an even integer whose definition is conveniently postponed to $\S 5$; we may remark, however, that there is always more than one sheet.

The functions*

$$
y_{j}^{(m)}(t)=\alpha_{j m} \sigma_{j m}(\xi),
$$

where

$$
\begin{aligned}
& \sigma_{j m}(\xi) \equiv \xi^{n(j-m) / p} \sum_{l=0}^{\infty} \frac{\left(\frac{\xi}{n}\right)^{l n}}{\prod_{i=0}^{n-1} \Gamma\left(l+\lambda_{i j m}+1\right)}, \\
& \lambda_{i j m} \equiv \frac{j-i}{p}- \begin{cases}1, & i<m, \\
0, & i \geqq m,\end{cases} \\
& \alpha_{j m} \equiv p^{(n j+\nu m) / p} n^{n(m-j) / p_{K_{j}},}
\end{aligned}
$$

* The following remarks apply to formulas (5) et seq.

(1) If $m>j$ one of the gamma functions in the denominator of the term for $l=0$ is infinite, and the term is then defined to be zero, thus removing the apparent singularity.

(2) In this and all subsequent formulas involving a real positive constant to a real power, the real positive value is to be used.

(3) For purposes of calculation it might be desirable when $\nu<n-1$ to replace the $n$ gamma functions in the denominator by $\nu$ gamma functions of the same type in the numerator and a single gamma function in the denominator. This is possible by applying the multiplication theorem of Gauss. See Whittaker and Watson's Modern Analysis, $\$ 12 \cdot 15$, or Nielsen's Handbuch der Theorie der Gammafunktion, §6. 
and

$$
\kappa_{j} \equiv \frac{1}{j !} \prod_{i=0}^{n-1} \Gamma\left(\lambda_{i j 0}+1\right),
$$

are then single-valued* at every point of the $\xi$-surface (4). Furthermore, although under the transformation (3), corresponding to some point $t$ there may be several points on the $\xi$-surface, all with the same absolute value but with arguments differing by integral multiples of $2 \pi p / n$, the value of $y_{j}{ }^{(m)}(t)$ calculated from the formula (5) is the same for all these points $\xi$.

The series (5) in ascending powers of $\xi$ converge very slowly for large $\xi$. It is therefore natural to seek series in descending powers to satisfy the $t$-equation. Let $\omega_{0}, \omega_{1}, \cdots, \omega_{n-1}$ be the $n$th roots of unity, and define

$$
\beta \equiv \frac{n-1}{2} .
$$

Then by formally differentiating the series

$$
s_{k}(t) \risingdotseq \frac{e^{\omega_{k} \xi}}{\xi^{\nu \beta / p}}\left\{1+\frac{b_{k 01}}{\xi}+\frac{b_{k 02}}{\xi^{2}}+\cdots\right\} \quad(k=0,1, \cdots, n-1)
$$

with respect to $t$,

$$
\begin{aligned}
s_{k}^{(m)}(t)=\omega_{k}^{m}\left(\frac{p}{n}\right)^{m \nu / p} \frac{e^{\omega_{k} \xi}}{\xi^{\nu(\beta-m) / p}}\left\{1+\frac{b_{k m 1}}{\xi}+\frac{b_{k m 2}}{\xi^{2}}+\cdots\right\} & \\
& (m=0,1, \cdots, n),
\end{aligned}
$$

and substituting in the $t$-equation, one finds that the constants $b_{k 0 l}$ can be successively determined and are unique. However, the series (6) are in general divergent and are hence called formal solutions.

Beginning with Poincaré's famous paper† in the Acta Mathematica in 1886 , and culminating in a recent paper by Trjitzinsky $\ddagger$ in the same journal, much progress has been made on the problem of showing that true solutions of a linear differential equation exist which under certain conditions are asymptotic to the formal solutions. The latter writer shows that for a very inclusive class of equations the neighborhood of $\infty$ can be completely covered by a finite number of regions, associated with each of which there exists a full set of true solutions uniformly asymptotic in the region to the formal solutions.

* It is to be understood here and hereafter that if $\arg Z$ is specified, the value to be chosen for $\arg Z^{\mu}, \mu$ real, is $\mu \arg Z$.

$\dagger$ Loc. cit.

$\ddagger$ Loc. cit. 
It now becomes necessary to digress for a moment in order to subdivide the $\xi$-surface. The rays $L_{\tau}$ from the origin,

$$
L_{\tau}: \quad \arg \xi=\theta_{\tau} \equiv \tau \frac{\pi}{n} \quad(\tau=0, \pm 1, \pm 2, \cdots, \pm Q),
$$

divide it into $2 Q$ sectors $S_{\tau}$,

$S_{\tau}$ :

$$
\theta_{\tau-1} \leqq \arg \xi \leqq \theta_{\tau}, \quad-Q+1 \leqq \tau \leqq Q .
$$

Although the system of $S$-sectors suffices for most of the discussion, a different system of sectors covering the $\xi$-surface is required to simplify the statement of our final results: Each of the rays $L_{2 q+1}, q$ integral, $-Q<2 q+1<Q$, is inclosed in a sector $D_{q, q+1}$ whose central angle $2 \delta$ is small compared with $\pi / n$,

$D_{q, q+1}$ :

$$
\theta_{2 q+1}-\delta \leqq \arg \xi \leqq \theta_{2 q+1}+\delta .
$$

The region between two successive $D$-sectors, and having as its bisector the line $L_{2 q}, q=0, \pm 1, \pm 2, \cdots, \pm(Q / 2-1)$, is denoted by $T_{q}$,

$$
T_{q}: \quad \theta_{2 q-1}+\delta \leqq \arg \xi \leqq \theta_{2 q+1}-\delta .
$$

The "left-over" regions are then called $T_{Q / 2}$ and $T_{-Q / 2}$,

$T_{Q / 2}$ :

$$
\theta_{Q-1}+\delta \leqq \arg \xi \leqq \theta_{Q},
$$

$T_{-Q / 2}$ :

$$
\theta_{-Q} \leqq \arg \xi \leqq \theta_{-Q+1}-\delta .
$$

All the $S, D$, and $T$ regions are closed except at $\xi=\infty$.

The fundamental theorem* of Trjitzinsky's paper applied to the $t$-equation insures the existence of true solutions and their $t$-derivatives, $\left\{{ }_{\tau} \tilde{y}_{k}^{(m)}(t)\right\}$, $k, m=0,1, \cdots, n-1$, analytic in $t$, such that

$$
{ }_{r} \tilde{y}_{k}^{(m)}(t) \sim s_{k}^{(m)}(t)
$$

uniformly with respect to $\xi$ for $\xi$ in $S_{\tau}$, that is,

$$
{ }_{{ }_{r}} \tilde{y}_{k}^{(m)}(t)=\omega_{k}^{m}\left(\frac{p}{n}\right)^{m \nu / p} \frac{e^{\omega_{k} \xi}}{\xi^{\nu(\beta-m) / p}}\left\{1+\frac{b_{k m 1}}{\xi}+\cdots+\frac{b_{k, m, \mu-1}}{\xi^{\mu-1}}+\frac{{ }_{r} b_{k m \mu}(\xi)}{\xi^{\mu}}\right\},
$$

where $\left|{ }_{\tau} b_{k m \mu}(\xi)\right|<M_{\mu}$ for $|\xi| \geqq N, \xi$ in $S_{\tau}, \mu=1,2,3, \cdots$.

4. The Stokes multipliers. Corresponding to a region $S_{\tau}$, the subscripts $k$ of the $n$th roots of unity $\left\{\omega_{k}\right\}$ can be arranged so that the following ordering of the real parts of $\left\{\omega_{k} \xi\right\}$ is maintained for $\xi$ in $S_{\tau}$ :

* Loc. cit., p. 208. For $n>2$ our regions $S_{i}$ are identical with Trjitzinsky's regions $R_{i}$ and require no further subdivision into regions $R_{i}^{\prime}$ and $R_{i}{ }^{\prime \prime}$ For $n=2$ the Trjitzinsky regions $R_{i}$ consist each of two of our quadrants $S_{\tau}$, but in this case subdivision is necessary into $R_{i}^{\prime}$ and $R_{i}{ }^{\prime \prime}$, each of which may be taken as a quadrant $S_{\boldsymbol{r}}$. 


$$
R\left(\omega_{0} \xi\right) \geqq R\left(\omega_{1} \xi\right) \geqq \cdots \geqq R\left(\omega_{n-1} \xi\right) .
$$

We shall call ${ }_{\tau} \tilde{y}_{0}$ the dominant solution and ${ }_{\tau} \tilde{y}_{1}$ the first sub-dominant solution in $S_{\tau}$. The actual values of $\omega_{0}$ and $\omega_{1}$ for each of the regions $S_{\tau}$ are given in the table at the end of this section.

Between the solutions $\left\{r_{r} \tilde{y}_{k}\right\}$ and the principal solutions $\left\{y_{j}\right\}$ a nonsingular linear relationship must exist,

$$
y_{j}=\sum_{k=0}^{n-1}{ }_{\tau} c_{j k}{ }_{\tau} \tilde{y}_{k} .
$$

It is of the greatest importance for us to evaluate the Stokes multipliers $\left\{{ }_{r} c_{j k}\right\}$, or at least the multipliers ${ }_{\tau} c_{j 0}$ and ${ }_{r} c_{j 1}$ of the dominant and first subdominant solutions. To accomplish this we utilize* a formula of Stokes.

By an ingenious method Stokes showed $\dagger$ that a power series whose terms are of the type

$$
X_{l}=\frac{\prod_{i=1}^{r} \Gamma\left(l+a_{i}+1\right)}{\prod_{i=1}^{s} \Gamma\left(l+b_{i}+1\right)} x^{l}, \quad \text { where }\left\{\begin{array}{l}
\mu \equiv s-r>0, \\
a_{i}, b_{i}, \text { real }, \\
a_{i} \neq-1,-2,-3, \cdots,
\end{array}\right.
$$

can be asymptotically summed for real positive $x$ by the formula

$$
\sum_{l=0}^{\infty} X_{l} \cong \frac{e^{\mu \chi}}{\left|\chi^{\alpha}(2 \pi \chi)^{(\mu-1) / 2} \mu^{1 / 2}\right|},
$$

where

$$
\begin{aligned}
& \alpha \equiv \sum_{i} b_{i}-\sum_{i} a_{i}, \\
& \chi \equiv\left|x^{1 / \mu}\right|,
\end{aligned}
$$

and the notation $y \cong u$ means $\lim _{x \rightarrow \infty}(y / u)=1$.

If we denote by $f_{j}(\xi)$ the series on the right side of equation (5) for $m=0$, so that

$$
y_{j}(t)=\alpha_{j 0} \xi^{n j / p} f_{j}(\xi),
$$

we see that $f_{j}(\xi)$ is directly amenable to the application of Stokes' formula with $x=(\xi / n)^{n}$. The result

* For the suggestion of this fundamental idea, as well as for other helpful advice, the author is indebted to Professor R. E. Langer.

$\dagger$ Note on the determination of arbitrary constants, etc., Proceedings of the Cambridge Philosophical Society, vol. 6 (1889), p. 362. The formula can also be found in Bromwich, An Introduction to the Theory of Infinite Series, London, 1926, Chapter XII, \$112. 


$$
f_{j}(\xi) \cong \frac{e^{|\xi|}}{(2 \pi)^{\beta} n^{1 / 2}}\left|\left(\frac{\xi}{n}\right)^{-(n j+\nu \beta) / p}\right|
$$

is then valid for $x$ real and positive, that is, for $\xi$ on $L_{2 q}, q=0, \pm 1, \pm 2, \cdots$, $\pm Q / 2$.

After eliminating the absolute value signs by means of the identity

$$
\left|\xi^{\alpha}\right| \equiv \frac{\xi^{\alpha}}{e^{i \alpha \arg \xi}}=e^{-2 \pi i \alpha q / h \xi^{\alpha}}
$$

on $L_{2 q}$, we obtain

$$
y_{j}(t) \cong{ }_{q} \gamma_{j} \frac{e^{\omega q \xi}}{\xi^{\nu \beta / p}}
$$

on $L_{2 q}$, where

and

$$
{ }_{q} \gamma_{j} \equiv \frac{\kappa_{j} p^{n j / p} e^{(2 \pi i q / n)(n j+\nu \beta) / p}}{(2 \pi)^{\beta} n^{1 / 2-\nu \beta / p}}
$$

$$
\omega \equiv e^{-2 \pi i / n} .
$$

We shall now make the result (9) from Stokes' formula yield us the multipliers ${ }_{r} c_{j 0}$ and ${ }_{r} c_{j 1}$.

First, we show that in any region $S_{\tau}$ which abuts on a ray $L_{2 q}$, the multiplier ${ }_{r} c_{j 0}$ of the dominant solution is the constant ${ }_{q} \gamma_{j}$ just found. The result (9) may be written

$$
y_{i}={ }_{a} \gamma_{j} \frac{e^{\omega} Q_{\xi \xi}}{\xi^{\nu \beta / p}}\{1+\epsilon(\xi)\},
$$

where $\epsilon(\xi) \rightarrow 0$ as $\xi \rightarrow \infty$ on $L_{2 q}$. Since $L_{2 q}$ is in $S_{\tau}$, the asymptotic formulas (7) are valid:

$$
y_{j}=\sum_{k=0}^{n-1}{ }_{\tau} c_{j k} \frac{e^{\omega_{k} \xi}}{\xi^{\nu \beta / p}}\left\{1+\frac{E(\xi)}{\xi}\right\}
$$

for $|\xi| \geqq N$; here $E(\xi)$ is a generic notation for functions of $\xi$, bounded for all $\xi$. Equating these expressions, and noting that $\omega_{0}=\omega^{q}$ in $S_{\tau}$, we obtain

$$
\left|{ }_{r} c_{j 0}\left\{1+\frac{E(\xi)}{\xi}\right\}-{ }_{a} \gamma_{j}\{1+\epsilon(\xi)\}\right|=\left|\sum_{k=1}^{n-1}{ }_{r} c_{j k} e^{\omega_{k} \xi-\omega_{0} \xi}\left\{1+\frac{E(\xi)}{\xi}\right\}\right| .
$$

Since on $L_{2 q}$

$$
R\left(\omega_{k} \xi-\omega_{0} \xi\right)=h_{k}|\xi|, \quad h_{k}<0, \quad k \neq 0,
$$


it follows, on letting $\xi \rightarrow \infty$ on $L_{2 q}$, that

$$
{ }_{r} c_{j 0}={ }_{q} \gamma_{j} .
$$

We have thus determined the multipliers of the dominant solutions in all the regions $S_{\tau}$.

Next, we observe that for two regions $S_{2 q}$ and $S_{2 q-1}$ which abut along a ray $L_{2 q-1},-Q<2 q-1<Q$, the values for $\omega_{0}$ and $\omega_{1}$ simply interchange as we cross $L_{2 q-1}$ :

$$
\begin{aligned}
& \omega_{0} \text { of } S_{2 q}=\omega_{1} \text { of } S_{2 q-1}=\omega^{q}, \\
& \omega_{1} \text { of } S_{2 q}=\omega_{0} \text { of } S_{2 q-1}=\omega^{q-1} .
\end{aligned}
$$

We are then able to prove that the multiplier of the first sub-dominant solution on one side of $L_{2 q-1}$ is the same as the multiplier of the dominant solution on the other side of $L_{2 q-1}$, and this latter we know. Equating

$$
y_{j}=\sum_{k=0}^{n-1} 2 q-1 c_{j k}{ }_{2 q-1} \tilde{y}_{k}=\sum_{k=0}^{n-1}{ }_{2 q} c_{j k 2 q} \tilde{y}_{k},
$$

and utilizing the asymptotic formulas (7), which are valid on $L_{2 q-1}$ for $\left\{2 q-1 \tilde{y}_{k}\right\}$ and $\left\{{ }_{2 q} \tilde{y}_{k}\right\}$, we obtain as before

$$
\begin{aligned}
& \mid\left\{{ }_{2 q-1} c_{j 0}\left[1+\frac{E(\xi)}{\xi}\right]-{ }_{2 q} c_{j 1}\left[1+\frac{E(\xi)}{\xi}\right]\right\} \\
& \quad+e^{\omega \alpha_{\xi-\omega} \sigma^{q-1} \xi}\left\{{ }_{2 q-1} c_{j 1}\left[1+\frac{E(\xi)}{\xi}\right]-{ }_{2 q} c_{j 0}\left[1+\frac{E(\xi)}{\xi}\right]\right\} \mid \rightarrow 0
\end{aligned}
$$

as $\xi \rightarrow \infty$ on $L_{2 q-1}$. Now

$$
R\left(\omega^{q \xi}-\omega^{q-1} \xi\right)=0
$$

on $L_{2 q-1}$, and therefore

$$
\omega^{q \xi}-\omega^{q-1} \xi=i h|\xi|,
$$

where $h$ is real and not zero. But if

$$
A(\xi)+e^{i h|\xi|} B(\xi) \rightarrow 0
$$

as $\xi \rightarrow \infty$ and

$$
A(\xi) \rightarrow A, \quad B(\xi) \rightarrow B,
$$

then

$$
A=0 \text { and } B=0 .
$$


Hence

$$
{ }_{2 q-1} c_{j 0}={ }_{2 q} c_{j 1} \text { and }{ }_{2 q-1} c_{j 1}={ }_{2 q} c_{j 0} .
$$

The results of this section for a region $S_{\tau}$ are summarized in the following table:

\begin{tabular}{|c|c|c|c|c|}
$\tau$ & $\omega_{0}$ & $\omega_{1}$ & ${ }_{{ }} c_{j 0}$ & ${ }_{{ }} c_{j 1}$ \\
\hline \hline $2 q$ & $\omega^{q}$ & $\omega^{q-1}$ & ${ }_{q} \gamma_{j}$ & ${ }_{q-1} \gamma_{j}$ \\
\hline $2 q+1$ & $\omega^{q}$ & $\omega^{q+1}$ & ${ }_{q} \gamma_{j}$ & ${ }_{q+1} \gamma_{j}$ \\
\hline
\end{tabular}

At this stage the asymptotic description of our special case is available. Instead of pausing for this now, however, we shall proceed to attack the general case on the basis of the results now at hand. After the treatment of the general case has been completed, results for the special case, if desired, can obviously be obtained by specialization.

\section{Chapter III. A Related equation}

5. The auxiliary variable $\xi$. Although the solutions discussed in this paper are known to be single-valued in $z$ and $\rho$ from the general existence theorem, our asymptotic formulas involve quantities multi-valued* in $z$ and $\rho$, and for this reason we introduce cuts in the $z$ - and $\rho$-planes.

The function $\phi(z) \equiv z^{\nu / n} \phi_{1}(z)$, in general not single-valued, $\dagger$ can be made so with the aid of a cut in the z-plane from the origin to the boundary of $R_{\mathbf{z}}$. The most convenient location of this cut will be specified later. Suppose tentatively some such cut, say that associated with the inequality

$$
-\pi<\arg z \leqq \pi
$$

With this convention the integral

$$
\Phi(z) \equiv \int_{0}^{z} \phi(z) d z
$$

* It is the essential multi-valuedness of these quantities that gives birth to the Stokes phenomenon when $\nu>0$ : Although one selects a definite branch of the multi-valued function, this eventually leads into another branch, and a change of coefficient becomes necessary to compensate for the change in the function. When $\nu=0$, the quantities are single-valued, and there is no Stokes phenomenon.

$\dagger$ For any given equation $\phi_{1}(z)$ may be chosen as an analytic function in $n$ different ways, since only $\phi_{1}^{n}(z)$ enters the equation through hypothesis (iii). It is assumed that a definite choice is now made, and adhered to henceforth. If $n$ divides $\nu, \phi(z)$ is then single-valued, but we make the cut nevertheless. 
becomes single-valued and independent of path. It is evidently of the form

$$
\Phi(z)=z^{p / n} \Phi_{1}(z),
$$

where $\Phi_{1}(z)$ is analytic in $R_{z}$ and $\Phi_{1}(0) \neq 0$. The following hypothesis is indispensable:

(iv) $\Phi_{1}(z) \neq 0$ in $R_{z}$.

We now continuously deform the cut in the z-plane into the curve

$$
\arg \Phi(z)=\frac{p}{n} \pi ;
$$

the latter is continuous with continuously turning tangent and does not cross itself. The correspondence between points $\Phi$ and $z$ established by equation (11) can now be made one-to-one by using in the $\Phi$-plane a Riemann surface with branch point at the origin, in the form of a sector of central angle $2 \pi p / n$ :

$$
-\frac{p}{n} \pi<\arg \Phi \leqq \frac{p}{n} \pi .
$$

The mapping of the z-plane on the $\Phi$-plane is then conformal except at the origin, where angles are multiplied by $p / n$.

From the $\rho$-plane we delete a small circle about the origin and then cut it along a ray:*

$$
|\rho| \geqq h>0, \quad-\pi<\arg \rho \leqq \pi .
$$

As in Langer's work on the second-order case, we find that the key to the problem of stating in the simplest way the asymptotic formulas and their validity lies in the consideration of an auxiliary variable $\xi$, where we define

$$
\xi \equiv \rho \Phi(z) .
$$

The $\Phi$-plane is thus mapped on the $\xi$-plane by a simple magnification and rotation of amount determined by $\rho$.

In order to transform the $t$-equation into a related equation of the same type as the given equation, we now identify this auxiliary variable $\xi$ with the variable $\xi$ already introduced in $\$ 3$ in connection with the $t$-equation. With the following definition of the number $Q$ of (4):

$$
Q \equiv 2 n+\nu+ \begin{cases}0, & \nu \text { even } \\ 1, & \nu \text { odd }\end{cases}
$$

\footnotetext{
* The region (12) does not include the point $\rho=\infty$.
} 
we see that we have achieved a construction by which, in accordance with equation (11) and (13), there corresponds to any pair of points respectively in $R_{z}$ and the $\rho$-plane a single point in the $\xi$-surface. At the same time we have rendered single-valued the quantities $\rho^{n / p}, \xi^{\nu / p}$, and $\xi^{\nu / p}$, which enter into our asymptotic formulas.

6. Transformation of $t$-equation into related equation. Suppose we define $v(z, \rho)$ from the equation

$$
y(t)=\psi^{\beta}(z) v(z, \rho),
$$

where $y(t)$ is any solution of the $t$-equation, and the variables $t$ and $z$ are connected by equations (3) and (13). We shall prove that with proper definition of $\psi(z)$, the function $v(z, \rho)$ satisfies a related equation in $R_{z}$.

We define

$$
\psi(z) \equiv \frac{\phi(z)}{\Phi^{\nu / p}(z)} .
$$

Since $\arg \Phi$ is single-valued on the $\Phi$-surface, $\psi(z)$ is single-valued in $R_{z}$. The apparent singularity at $z=0$ is removed by the otherwise equivalent expression

$$
\psi(z)=\frac{\phi_{1}(z)}{\Phi_{1}{ }^{1 / p}(z)},
$$

which shows that $\psi(z)$ and also $\psi^{-1}(z)$ are analytic in $R_{z}$. Furthermore, since $\psi(z)$ is bounded from zero in $R_{z}$, it is possible to make a single-valued determination of $\psi^{\beta}(z)$ even when $\beta=(n-1) / 2$ is not integral, and this will be analytic in $R_{\boldsymbol{z}}$.

Successive differentiations* of the above equation yield

$$
y^{(m)}(t)=\rho^{-m n / p}\left(\frac{p}{n}\right)^{m \nu / p} \psi^{\beta}(z) \sum_{j=0}^{m} g_{m j}(z) v^{(j)}(z, \rho),
$$

where $g_{m j}(z)$ is a polynomial in $\psi^{-1} ; \psi^{\prime}, \psi^{\prime \prime}, \cdots, \psi^{(n)}$ for $m \leqq n$, and is consequently analytic in $R_{z}$; in particular,

$$
\begin{aligned}
g_{m m}(z) & =\psi^{-m}(z), \\
g_{m, m-1}(z) & =\frac{m}{2}(n-m) \psi^{-m-1}(z) \psi^{\prime}(z) .
\end{aligned}
$$

Substituting in the $t$-equation, we obtain the related equation

\footnotetext{
* The differentiations indicated by the superscripts are with respect to $t$ on the left side of the equations and $z$ on the right side.
} 


$$
v^{(n)}(z, \rho)+0+g_{2}(z) v^{(n-2)}(z, \rho)+\cdots+\left\{g_{n}(z)-\rho^{n} \phi^{n}(z)\right\} v(z, \rho)=0,
$$

where

$$
g_{j}(z) \equiv \psi^{n}(z) g_{n j}(z)
$$

is analytic in $R_{\mathbf{z}}$.

The linear system (15), $m=0,1, \cdots, n-1$, can be inverted,

$$
v^{(m)}(z, \rho)=\psi^{-\beta}(z) \sum_{j=0}^{m} \rho^{j n / p}\left(\frac{n}{p}\right)^{j \nu / p} \hat{g}_{m j}(z) y^{(j)}(t),
$$

the coefficients $\hat{g}_{m j}(z)$ being analytic in $R_{z}$, and in particular,

$$
\hat{g}_{m m}(z)=\psi^{m}(z) .
$$

Those solutions of the related equation which correspond to $y(t)={ }_{\tau} \tilde{y}_{k}(t)$ we shall denote by

$$
{ }_{\tau} \tilde{v}_{k}(z, \rho) \equiv \psi^{-\beta}(z){ }_{\tau} \tilde{y}_{k}(t) .
$$

Since ${ }_{\tau} y_{k}^{(j)}(t)$ is analytic in

$$
t=\rho^{n / p} z\left\{\frac{p}{n} \Phi_{1}(z)\right\}^{n / p}
$$

it follows from (17) that ${ }_{\tau} \tilde{v}_{k}^{(m)}(z, \rho)$ is an analytic function of $z$ in $R_{z}$ and of $\rho$ in the region (12).

In the next chapter we shall need the asymptotic formulas

$$
\tau_{\tau} \tilde{v}_{k}^{(m)}(z, \rho)=\rho^{m n / p} \psi^{m-\beta}(z)\left(\frac{n}{p}\right)^{m \nu / p}\left\{\tilde{y}_{k}^{(m)}(t)+\frac{E(z, \rho)}{\rho^{n / p}}\right\}
$$

for $|\xi|<N$, and

$$
\tilde{\tau} k^{(m)}(z, \rho)=\rho^{m n / p} \omega_{k}^{m} \psi^{m-\beta}(z) \frac{e^{\omega_{k} \xi}}{\xi^{\nu(\beta-m) / p}}\left\{1+\frac{E(\xi)}{\xi}+\frac{E(z, \rho)}{\rho^{n / p}}\right\}
$$

for $\xi$ in $S_{\tau}$ and $|\xi| \geqq N$. Here as elsewhere in this paper the symbol $E$ denotes a function of the indicated variables bounded for all values of those variables. The first of the formulas follows immediately from equation (17) and the fact that ${ }_{r} y_{k}^{(j)}(t)$, being a linear combination of $\sigma_{r j}(\xi)$ (see (5) et seq.) with constant coefficients, is bounded for $|\xi|<N$. The second follows from (17) together with the asymptotic expressions (7).

\section{Chapter IV. Comparison OF SOlutions}

7. An equivalent integral equation. In this chapter we shall compare certain solutions of the given and related equations by an appropriate modification of a method of Birkhoff.*

* On the asymptotic character, etc., loc. cit., especially p. $226 \mathrm{ff}$. 
Since the index $\tau$ is fixed for the course of the chapter, we shall drop it from the symbols $\left\{{ }_{r} v_{k}^{(m)}(z, \rho)\right\}$. If $\left\{w_{j}(z, \rho)\right\}$ are defined from the equations*

$$
\sum_{j=0}^{n-1} \tilde{v}_{j}^{(m)}(z, \rho) w_{j}(z, \rho)=\delta_{m, n-1} \quad(m=0,1, \cdots, n-1),
$$

it is easily seen that they are analytic functions of $z$ in $R_{z}$ and of $\rho$ in (12). An integral equation equivalent to the given equation (1) is

$$
u(z, \rho)=\sum_{j=0}^{n-1} c_{j} \tilde{v}_{j}(z, \rho)+\int_{0}^{z}\left\{\sum_{j=0}^{n-1} \tilde{v}_{j}(z, \rho) w_{j}(\zeta, \rho)\right\} L[u(\zeta, \rho)] d \zeta,
$$

where

$$
L[u(\zeta, \rho)] \equiv \sum_{j=0}^{n-2}\left\{g_{n-j}(\zeta)-p_{n-j}(\zeta)\right\} u^{(j)}(\zeta, \rho),
$$

and $\left\{c_{j}\right\}$ is a set of arbitrary constants (i.e., functions of $\rho$ alone). If we contemplate only solutions of (20) analytic in $R_{z}$, the path of integration is evidently immaterial as long as it lies in $R_{z}$.

If the set $\left\{c_{j}\right\}$ is fixed, the existence and uniqueness of the solution of (20) which is analytic in $z$ over $R_{z}$ follow from the fundamental existence theorem applied to (1); for the solution of (20) is identical with that solution of (1) which obeys the boundary conditions

$$
u^{(m)}(0, \rho)=\sum_{j=0}^{n-1} c_{j} \tilde{v}_{j}^{(m)}(0, \rho) \quad(m=0,1, \cdots, n-1) .
$$

This solution and its derivatives are analytic in $\rho$ if the constants $\left\{c_{j}\right\}$ are.

For the purpose of showing that to each solution $\tilde{v}_{k}(z, \rho)$ of the related equation there corresponds a solution $\tilde{u}_{k}(z, \rho)$ of the given equation of a certain type, the integral in equation (20) is not yet in tractable form. A suitable form of the integral equation for a definite $k$ is

$$
\begin{aligned}
u(z, \rho)=\sum_{j=0}^{n-1} c_{j}^{\prime} \tilde{v}_{j}(z, \rho) & +\int_{z}^{z}\left\{\sum_{j=0}^{k} \tilde{v}_{j}(z, \rho) w_{j}(\zeta, \rho)\right\} L[u(\zeta, \rho)] d \zeta \\
& +\int_{0}^{z}\left\{\sum_{j=k+1}^{n-1} \tilde{v}_{j}(z, \rho) w_{j}(\zeta, \rho)\right\} L[u(\zeta, \rho)] d \zeta
\end{aligned}
$$

where $Z$ is a fixed point of $R_{z}$; and this results from the following transformation of the constants:

${ }^{*} \delta_{i j}$ is the "Kronecker $\delta . "$ 


$$
\begin{array}{lrl}
c_{j}=c_{j}^{\prime}-\int_{0}^{z} w_{j}(\zeta, \rho) L[u(\zeta, \rho)] d \zeta & (j=0,1, \cdots, k), \\
c_{j}=c_{j}^{\prime} & (j=k+1, \cdots, n-1) .
\end{array}
$$

The solution* $\tilde{u}_{k}(z, \rho)$ is now defined to be the solution of (22) for $\left\{c_{j}^{\prime}=\delta_{j k}\right\}$. One would like to establish, for certain "admitted values" of $\arg \rho$ and $|\rho|>\rho_{1}$, the existence and uniqueness of this solution, together with its analyticity in $\rho$, after which it is easy to derive its asymptotic form. It is prerequisite, however, to inquire into the nature of suitable curves of integration and the existence of certain bounds. It is therefore convenient to conclude this section with a lemma to whose proof we can return after developing the necessary machinery.

From (21) we see that the solution of (20) determined by the set $\left\{c_{j}\right\}$ is

$$
u(z, \rho)=\sum_{j=0}^{n-1} c_{j} U_{j}(z, \rho),
$$

where $\left\{U_{j}(z, \rho)\right\}$ are the solutions of (1) with the boundary values

$$
U_{j}{ }^{(m)}(0, \rho)=\tilde{v}_{j}{ }^{(m)}(0, \rho) \quad(m=0,1, \cdots, n-1) .
$$

Hence

$$
c_{j}^{\prime}=\sum_{l=0}^{n-1} \gamma_{j l}(\rho) c_{l} .
$$

The coefficients $\gamma_{j l}(\rho)$ of this transformation are analytic in $\rho$, since the boundary values $\left\{\tilde{v}_{j}^{(m)}(0, \rho)\right\}$, and therefore the solutions $\left\{U_{j}(z, \rho)\right\}$, and, as we have seen, the functions $\left\{w_{j}(z, \rho)\right\}$, all have this property.

If the following lemma is true, then, for admitted values of $\arg \rho$ and $|\rho|>\rho_{1}$, a unique set $\left\{c_{j}\right\}$ analytic in $\rho$ will correspond to the set $\left\{c_{j}^{\prime}=\delta_{j k}\right\}$, and consequently by the statements associated with equation (21), we shall have established the existence, uniqueness, and analyticity of $\tilde{u}_{k}(z, \rho)$ for such $\rho$.

Leмma. There exists a number $\rho_{1}$ such that, for $|\rho|>\rho_{1}$ and admitted values of $\arg \rho$, the determinant $\left|\left(\gamma_{j l}(\rho)\right)\right|$ of the system (23) can have no zero.

8. Curves $\Gamma$, regions $r$, and configurations $C$. The quantity $\arg \rho$ now assumes critical significance, and it will simplify the notation to denote it by a single symbol $\lambda$,

$$
\lambda \equiv \arg \rho .
$$

* This solution is evidently a function of $Z$ and $\tau$; we shall, however, drop these prescripts from the more precise notation $z, r \tilde{u}_{k}(z, \rho)$ until we reach the existence theorem at the end of the chapter. 
By a rotation about the origin through an angle $-\lambda$, the complex of rays $L_{\tau}$ and sectors $S_{\tau}$ of the $\xi$-surface is mapped back on the $\Phi$-surface, where we designate it by (cf. $\$ 3$ )

$L_{\tau}(\lambda)$ :

$$
\arg \Phi=\theta_{\tau}-\lambda \equiv \theta_{\tau}(\lambda)
$$

and

$S_{r}(\lambda)$ :

$$
\theta_{\tau-1}(\lambda) \leqq \arg \Phi \leqq \theta_{\tau}(\lambda) .
$$

Suppose we call the map of $R_{z}$ on the $\Phi$-surface $R_{\Phi}$. With such parts of the complex of rays $L_{\tau}(\lambda)$ and regions $S_{\tau}(\lambda)$ as fall outside $R_{\Phi}$, we shall not be concerned.

Let $Z$ be a fixed point of $R_{z}$, not the origin. The corresponding point $\Phi(Z)$ in $R_{\Phi}$ then lies in some region $S_{\tau}(\lambda)$, where the subscript $\tau$ is a step function of $\lambda$. The following definitions are framed relative to some definite value of $\tau$.

$A$ curve $\Gamma_{Z, \tau}(\lambda)$ is defined to have the following properties:

[1] It is an ordinary curve connecting 0 and $Z$, lying in the cut region $R_{2}$.

A curve is here called ordinary if it is continuous and has a unique tangent everywhere except possibly at a finite number of points where there exist two tangents, one for each sense of approach. A positive sense of traversal of the curve can be established; we take this to be from 0 to $Z$. Now consider the map of $\Gamma_{Z, \tau}(\lambda)$ on $R_{\Phi}$. Let $\mathrm{t}$ be a unit vector directed along the tangent in the positive sense:

[2] At every point* on the curve

$$
\theta_{\tau-1}(\lambda) \leqq \arg t \leqq \theta_{\tau}(\lambda)
$$

for some determination of arg $t$.

In order to see the significance of property [2], suppose $z_{\alpha}$ and $z_{\beta}$ are two points on a $\Gamma$-curve. To signify that the curve is traversed in the positive sense in passing from $z_{\alpha}$ to $z_{\beta}$ we shall write

$$
z_{\alpha}<z_{\beta} \text {. }
$$

Let

$$
\xi_{j} \equiv \rho \Phi\left(z_{j}\right)
$$$$
(j=\alpha, \beta)
$$

The property [2] insures that if $z_{\alpha} \leqq z_{\beta}$,

$$
R\left[\omega_{0}\left(\xi_{\beta}-\xi_{\alpha}\right)\right] \geqq R\left[\omega_{1}\left(\xi_{\beta}-\xi_{\alpha}\right)\right] \geqq \cdots \geqq R\left[\omega_{n-1}\left(\xi_{\beta}-\xi_{\alpha}\right)\right] .
$$

* At an exceptional point both values of $t$ must satisfy [2]. 
For $n=2$, the property [2] can be replaced* by the less stringent

$$
\begin{aligned}
& \theta_{\tau-1}(\lambda) \leqq \arg \mathrm{t} \leqq \theta_{\tau+1}(\lambda), \quad \tau \text { even }, \\
& \theta_{\tau-2}(\lambda) \leqq \arg \mathrm{t} \leqq \theta_{\tau}(\lambda), \quad \tau \text { odd } ;
\end{aligned}
$$

together with

[3] The map in $R_{\Phi}$ of $\Gamma_{z, \tau}(\lambda)$ lies entirely in $S_{\tau}(\lambda)$; and

[4] There exists a number $G$, independent of $Z, \tau, \lambda$, such that the length of all curves $\Gamma_{Z, \tau}(\lambda)$ is $<G$.

The properties [3] and [4] are implied by [2].

$A$ region $r_{Z, \tau}(\lambda)$ in $R_{z}$ is defined to be a simply connected region containing 0 and $Z$, every point of which lies on some curve $\Gamma_{z, \tau}(\lambda)$.

Although $L_{\tau}(\lambda)$ and $S_{\tau}(\lambda)$ are originally constructed on $R_{\Phi}$, and $\Gamma_{z, \tau}(\lambda)$ and $r_{Z, r}(\lambda)$ on $R_{z}$, no confusion will result from designating the curve or region and its map on the other surface by the same symbol.

Evidently $r_{z, r}(\lambda)$ lies completely in $S_{r}(\lambda)$.

The method of comparison employed is valid for a region of the type $r_{Z, \tau}(\lambda)$. It is hence important to secure that, for each $\lambda$ under consideration, every point of $R_{z}$ lies in some $r_{z, r}(\lambda)$. Since it may be possible to accomplish this for the desired $R_{z}$ only for a range of $\lambda$ more restricted than (12), we shall refer to admitted values of $\lambda$. Necessary and sufficient is hypothesis

(v) For each admitted value of $\lambda \equiv \arg \rho$, every point $z \neq 0$ in $R_{z}$ can be connected to the origin by a curve $\Gamma_{z, \tau}(\lambda)$.

The condition is necessary; for, if $z$ lies in some $r_{Z, \tau}(\lambda)$, it lies on some curve $\Gamma_{z, \tau}(\lambda)$. The portion of the curve between 0 and $z$ will serve as $\Gamma_{z, \tau}(\lambda)$. On the other hand, suppose $z$ lies on $\Gamma_{z, \tau}(\lambda)$. Take $Z=z$; then $z$ lies in an $r_{Z, \tau}(\lambda)$ consisting at least of this curve. Thus, the condition is also sufficient. $A$ configuration $C_{Z ; \tau}$ consists, for fixed $Z, \tau$, of all pairs of values $(z, \rho)$ such that for each admitted $\lambda \equiv \arg \rho$ the corresponding $z$ lies in $r_{Z, \tau}(\lambda)$.

To clarify the nature of a configuration $C_{Z, \tau}$, suppose $\lambda_{0}$ is an admitted value of $\lambda$, and $Z$ any point of $R_{z}$ not 0 , henceforth held fixed. By the above hypothesis $\Phi(Z)$ lies on some curve $\Gamma_{Z, \tau}\left(\lambda_{0}\right)$. If $n>2$ the region $r_{Z, \tau}\left(\lambda_{0}\right)$ as seen on $R_{\Phi}$ will then consist at most of the parallelogram with vertices at 0 and $\Phi(Z)$ and a pair of sides along $L_{\tau-1}\left(\lambda_{0}\right), L_{\tau}\left(\lambda_{0}\right)$. This maximum extent of

\footnotetext{
* The anomaly of the second-order case is related to the following simple geometrical problem: A circle and a straight line lie in the same plane. The circle is divided into $n$ equal parts by the points $\bar{\omega}_{0}, \bar{\omega}_{1}, \cdots, \bar{\omega}_{n-1}$. These are projected orthogonally into the points $\alpha_{0}, \alpha_{1}, \cdots, \alpha_{n-1}$ on the line. What is the maximum range of rotation of the circle about its center under the condition that no two points $\alpha_{j}, \alpha_{k}$ are allowed to cross each other? The answer is $\pi / n$ for $n>2$, but $2 \pi / n$ for $n=2$.
} 
$r_{Z, \tau}\left(\lambda_{0}\right)$ will be possible if and only if the boundary of $R_{\Phi}$ does not cut across the parallelogram. The minimum extent of $r_{Z, \tau}\left(\lambda_{0}\right)$ will be the curve $\Gamma_{Z, \tau}\left(\lambda_{0}\right)$; in any case it lies entirely inside the closed region bounded by the parallelogram. All the pairs $(z, \rho)$ for which $z$ lies in $r_{Z, \tau}\left(\lambda_{0}\right)$ and arg $\rho=\lambda_{0}$ will form a subset $C_{Z, \tau}\left(\lambda_{0}\right)$ of $C_{Z, \tau}$. If we now permit $\lambda$ to vary from $\lambda_{0}$, the bounding parallelogram changes shape, the vertices $0, \Phi(Z)$ remaining fixed, as well as the angles, but the sides rotating with the complex of rays $L_{q}(\lambda)$. There will evidently exist an interval $\lambda_{1}, \lambda_{1}+\pi / n$ in which the parallelogram varies between limiting positions in the form of the line segment from 0 to $\Phi(Z)$, and beyond which $\lambda$ can not vary without changing the $\tau$ of our definitions. For each admitted $\lambda$ in the interval, the above discussion for $\lambda=\lambda_{0}$ is valid and a subset $C_{Z, \tau}(\lambda)$ of $C_{Z, \tau}$ is generated. The sum of the subsets $C_{Z, \tau}(\lambda)$ for all admitted $\lambda$ in the interval is identical with $C_{\boldsymbol{Z}, \boldsymbol{r}}$.

The bounding parallelogram maps conformally on $R_{z}$ except at the origin where the angle $\pi / n$ between the sides maps into an angle $\pi / p$ between the corresponding curvilinear segments.

Because of the rotational symmetry about the origin, of the definitions with respect to integral multiples of the angle $\pi / n$, it follows that if $\lambda_{0}$ is an admitted value, all values $\lambda \equiv \lambda_{0}(\bmod \pi / n)$ in the interval (12) are also admissible. In particular, if the whole interval $\lambda_{1}, \lambda_{1}+\pi / n$ is admitted, all of (12) is admissible. In this case $R_{\Phi}$ is characterized by the property that no ray from the origin re-enters $R_{\Phi}$ after once leaving it. This follows from the nature of the limiting positions of the bounding parallelograms.

If we define the curves $\Gamma$ from the property [2] in the case $n=2$, the above discussion applies. If, however, we use $\left[2^{\prime}\right],[3],[4]$, the shape of the regions $r_{Z, \tau}(\lambda)$ is much more general. The implications of [1], [3], [4] are evident; [2'] implies the following characteristic: A region $r_{Z, \tau}(\lambda)$ has in common with any parallel to $L_{1}(\lambda)$ at most one segment (possibly a point). The modulus of congruence of the admitted values of $\lambda$ then becomes $\pi$ instead of $\pi / n$. However, the shape of an $R_{\Phi}$ valid for all $\lambda$ in (12) is of the same type as above.

9. Calculation of bounds. Because the solutions are of two distinctly different types according as $|\xi|<N$ or $|\xi| \geqq N$, we find it useful to define a function $s$ as follows:

Write

$$
s \equiv\left\{\begin{array}{l}
0,|\xi|<N \\
1,|\xi| \geqq N
\end{array}\right.
$$

$$
\tilde{v}_{j}^{(m)}(z, \rho)=\frac{\rho^{m n / p} e^{\omega_{j} \xi}}{\xi^{s \nu(\beta-m) / p}} \hat{v}_{j m}(z, \rho)(j, m=0,1, \cdots, n-1)
$$


From the formulas (18) and (19) we have for $\rho$ in (12) and $\xi$ in $S_{\tau}$,

$$
\left|\hat{v}_{j m}(z, \rho)\right|<M_{1} \text {. }
$$

Again, if we write

$$
w_{j}(z, \rho)=\frac{\hat{w}_{j}(z, \rho)}{\rho^{2 \beta n / p} \xi^{8 \nu \beta / p} e^{\omega_{j} \xi}},
$$

we see that the $\left\{\hat{w}_{j}(z, \rho)\right\}$ are determined from the equations

$$
\sum_{j=0}^{n-1} \hat{v}_{j m}(z, \rho) \widehat{w}_{j}(z, \rho)=\delta_{m, n-1} \quad(m=0,1, \cdots, n-1) .
$$

By solving (25) for the element $\hat{v}_{j m}(z, \rho)$ and utilizing the formula (19), the determinant $\Delta(z, \rho)$ of this system is obtained in the form

$$
\Delta(z, \rho)=\left|\left(\omega_{j}{ }^{m}\right)\right|+\frac{E(\xi)}{\xi}+\frac{E(z, \rho)}{\rho^{n / p}}
$$

for $|\xi| \geqq N$ and $\xi$ in $S_{\tau}$. The well known matrix $\left(\omega_{j}{ }^{m}\right), j, m=0,1, \cdots, n-1$, cannot have a zero determinant, and hence, after possibly necessary readjustment of the constant $N$ to a larger value, the determinant $\Delta(z, \rho)$ is bounded from zero for $|\rho|>$ some $\rho_{2}, \xi$ in $S_{\tau}$, and $|\xi| \geqq N$. A similar result can be obtained for $|\xi|<N$, since, by (18),

$$
\Delta(z, \rho)=\left(\frac{n}{p}\right)^{\nu n \beta / p} W\left[{ }_{\tau} \tilde{y}_{j}(t)\right]+\frac{E(z, \rho)}{\rho^{n / p}} .
$$

Here $W$ is the Wronskian of the solutions $\left\{_{\tau} \tilde{y}_{j}(t)\right\}$, and since these satisfy an equation in normal form, $W=$ constant, and since they are independent, $W \neq 0$. We thus conclude for $|\rho|>\rho_{3}$, and $\xi$ in $S_{\tau}$,

$$
\left|\hat{\hat{v}}_{j m}(z, \rho)\right|<M, \quad\left|\hat{w}_{j}(z, \rho)\right|<M .
$$

Finally, if we write

$$
\begin{aligned}
\sum_{j=0}^{k} \widehat{v}_{j}^{(m)}(z, \rho) w_{j}(\zeta, \rho) & \equiv \frac{\rho^{n(m-2 \beta) / p} e^{\omega_{k}(\xi-\bar{\xi})}}{\xi^{s \nu(\beta-m) / p \bar{\xi}^{s \nu}(\beta-m) / p}} A_{k m}(z, \zeta, \rho) \\
& \equiv F_{k m}(z, \zeta, \rho) A_{k m}(z, \zeta, \rho), \\
\sum_{j=k+1}^{n-1} \tilde{v}_{j}^{(m)}(z, \rho) w_{j}(\zeta, \rho) & \equiv F_{k m}(z, \zeta, \rho) B_{k m}(z, \zeta, \rho),
\end{aligned}
$$

where it is now necessary to distinguish between $\xi \equiv \rho \Phi(z)$ and $\bar{\xi} \equiv \rho \Phi(\zeta)$, likewise $s \equiv f(\xi)$ and $\bar{s} \equiv f(\bar{\xi})$, we can prove that the functions $A_{k m}$ and $B_{k m}$ are bounded in the following manner for $|\rho|>\rho_{3}$ and $\xi, \bar{\xi}$ in $S_{\tau}$ : 


$$
\begin{array}{ll}
\left|A_{k m}(z, \zeta, \rho)\right|<T, & 0 \leqq z \leqq \zeta \leqq Z, \\
\left|B_{k m}(z, \zeta, \rho)\right|<T, & 0 \leqq \zeta \leqq z \leqq Z,
\end{array}
$$

where $T$ is independent of $Z$. From (25), (26), (27),

$$
\left|\widetilde{v}_{j}^{(m)}(z, \rho) w_{j}(\zeta, \rho)\right|<\left|F_{j m}(z, \zeta, \rho)\right| M^{2} .
$$

But from (24) we know

$$
\left|F_{j m}(z, \zeta, \rho)\right| \leqq\left|F_{k m}(z, \zeta, \rho)\right|
$$

with the restriction

$$
\begin{array}{lr}
0 \leqq z \leqq \zeta \leqq Z & (j=0,1, \cdots, k), \\
0 \leqq \zeta \leqq z \leqq Z & (j=k+1, \cdots, n-1) .
\end{array}
$$

Combining these inequalities with (28), we have (29).

We are now equipped with the necessary bounds to prove our lemma. It is convenient, however, first to write the equation (22) and those resulting from differentiation of (22) in a form exhibiting the functions $\left\{a_{m}(z, \rho)\right\}$ which are defined from

$$
u^{(m)}(z, \rho) \equiv \frac{\rho^{m n / p} e^{\omega_{k} \xi}}{\xi^{s v(\beta-m) / p}} a_{m}(z, \rho) ;
$$

namely, with $m=0,1, \cdots, n-1$,

$$
\begin{aligned}
a_{m}(z, \rho)=\sum_{j=0}^{n-1} c_{j}^{\prime} e^{\left(\omega_{j}-\omega_{k}\right) \xi \hat{v}_{j m}}(z, \rho) & +\int_{z}^{z} A_{k m}(z, \zeta, \rho) \hat{L}[u(\zeta, \rho)] d \zeta \\
& +\int_{0}^{z} B_{k m}(z, \zeta, \rho) \hat{L}[u(\zeta, \rho)] d \zeta,
\end{aligned}
$$

where

$$
\hat{L}[u(\zeta, \rho)] \equiv \sum_{j=0}^{n-2} \frac{\left\{g_{n-j}(\zeta)-p_{n-j}(\zeta)\right\} a_{j}(\zeta, \rho)}{\rho^{n(2 \beta-j) / p \bar{\xi}^{\delta \nu}(2 \beta-j) / p}} .
$$

10. Proof of lemma; existence theorem. We now specify the path of integration to be a curve $\Gamma_{z, \tau}(\lambda)$ whenever $\lambda$ is an admitted value. Suppose for some $\rho$ with admitted $\lambda$ the determinant $\left|\left(\gamma_{j l}(\rho)\right)\right|$ has a zero. Then with $\left\{c_{j}^{\prime}\right\}$ all zero, a set $\left\{c_{j}\right\}$ not all zero can be found to satisfy (23). That is, for this $\rho$ there exists a solution $u(z, \rho) \not \equiv 0$ of the equation (22) with $\left\{c_{j}^{\prime}\right\}$ all zero.

Let $U(\rho) \neq 0$ be the maximum of $\left|\hat{a}_{m}(z, \rho)\right|, m=0,1, \cdots, n-1$, in the region $r_{z, \tau}(\lambda)$. Since the coefficients $g_{l}(z)$ and $p_{l}(z)$ are analytic in the closed region $R_{z}$, 


$$
\left|g_{l}(z)-p_{l}(z)\right|<D \quad(l=2,3, \cdots, n) ;
$$

and hence from (31), ${ }^{*}$

$$
\left|a_{m}(z, \rho)\right|<(n-1) \cdot T \cdot D \cdot G \cdot \frac{U(\rho)}{|\rho|^{n / p}} .
$$

But for some $m=m_{1}(\rho)$ and some $z=z_{1}(\rho)$,

$$
\frac{n-1}{n} U(\rho)<\left|a_{m}(z, \rho)\right| \text {. }
$$

Combining the last two inequalities, we see our lemma is_verified with

$$
\rho_{1} \equiv\{n \cdot T \cdot D \cdot G\}^{p / n} .
$$

With the machinery constructed to prove the lemma, we can easily derive the asymptotic forms of $\left\{\tilde{\tilde{u}}_{k}^{(m)}(z, \rho)\right\}$ for $(z, \rho)$ in the configuration $C_{Z, \tau}$. If the constants $\left\{c_{j}^{\prime}\right\}$ of $(22)$ are specified to be $\left\{\delta_{j k}\right\}$, the solution $u(z, \rho)$ becomes identical with $\tilde{u}_{k}(z, \rho)$,

$$
u^{(m)}(z, \rho) \equiv \tilde{u}_{k}^{(m)}(z, \rho) .
$$

With a definition of $U(\rho)$ analogous to the previous, we obtain from (31), for admitted $\lambda$,

$$
\left|a_{m}(z, \rho)\right|<M+(n-1) \cdot T \cdot D \cdot G \cdot \frac{U(\rho)}{|\rho|^{n / p}},
$$

and, as before, the left side may be replaced for some $z, m$ by $(n-1) U(\rho) / n$. Hence

$$
U(\rho)<\frac{2 n}{n-1} M
$$

for

$$
|\rho|>\rho_{0} \equiv 2^{p / n} \rho_{1} .
$$

We conclude now from (31) that, for $|\rho|>\rho_{0}$,

$$
\left|a_{m}(z, \rho)-\hat{v}_{k m}(z, \rho)\right|<\frac{2 n \cdot M \cdot T \cdot D \cdot G}{|\rho|^{n / p}} .
$$

On noting that this bound and $\rho_{0}$ are independent of $Z$, we are led to r-curves.

* Note $2 \beta-j \geqq 1$ if $j \leqq n-2$; also recall the property [4] that $G$ is a bound for the length of all 
THEOREM 1. Corresponding to any configuration $C_{Z, \tau}$ there exist $n$ solutions $\left\{z, \tau \tilde{u}_{k}(z, \rho)\right\}$ of the given equation, which, for $(z, \rho)$ in $C_{z, \tau}$ and $|\rho|>\rho_{0}$, are analytic in $\rho$ and satisfy the relations

$$
z, \tau \tilde{u}_{k}^{(m)}(z, \rho)={ }_{r} \tilde{v}_{k}^{(m)}(z, \rho)+\rho^{(m-1) n / p} E(z, \rho) \quad(k, m=0,1, \cdots, n-1)
$$

when $|\xi|<N$, and

$$
{ }_{z, \tau} \tilde{u}_{k}^{(m)}(z, \rho)=\rho^{m n / p} \omega_{k}^{m} \psi^{m-\beta}(z) \frac{e^{\omega_{k} \xi}}{\xi^{\nu(\beta-m) / p}}\left\{1+\frac{E(\xi)}{\xi}+\frac{E(z, \rho)}{\rho^{n / p}}\right\}
$$

when $|\xi| \geqq N$. Here the E-functions are bounded uniformly for all configurations $C_{Z, \tau}$.

The independence of these solutions is evident from their forms (33).

Chapter V. Boundary value Problem at the zero

11. Forms for the solutions principal at the zero. The solutions $\left\{u_{j}(z, \rho)\right\}$ of the given equation, analytic in $z$ and $\rho$, which satisfy the boundary conditions

$$
u_{j}^{(m)}(0, \rho)=\delta_{j m} \quad(j, m=0,1, \cdots, n-1),
$$

constitute the set we call principal at the origin. These must be linear combinations of the solutions described in Theorem 1,

$$
u_{j}(z, \rho)=\sum_{k=0}^{n-1}{ }_{z, \tau} \beta_{j k}(\rho){ }_{z, r} \tilde{u}_{k}(z, \rho) .
$$

In order to derive the form of ${ }_{Z, \tau} \beta_{j k}(\rho)$ for admitted $\lambda \equiv \arg \rho$ and $|\rho|>\rho_{0}$, let us hold $Z, \tau$ fixed until further notice. Meanwhile, we shall drop these subscripts wherever they occur.

For $|\xi|<N,(z, \rho)$ in the configuration $C$, and $|\rho|>\rho_{0}$, we have from (32)

where

$$
u_{j}{ }^{(m)}(z, \rho)=f_{m}(z, \rho) \sum_{k=0}^{n-1} \beta_{j k}(\rho)\left\{y_{k}{ }^{(m)}(t)+\frac{E(z, \rho)}{\rho^{n / p}}\right\}
$$

$$
f_{m}(z, \rho) \equiv \rho^{m n / p} \psi^{m-\beta}(z)\left(\frac{n}{p}\right)^{m \nu / p}
$$

Since $\left\{y_{j}\right\}$ and $\left\{\tilde{y}_{k}\right\}$ are each sets of $n$ independent solutions, the system (8) can be inverted,

$$
\tilde{y}_{k}=\sum_{l=0}^{n-1} \hat{\imath}_{k l} y_{l} \quad(k=0,1, \cdots, n-1),
$$


where

$$
\sum_{k=0}^{n-1} c_{j k} \hat{c}_{k l}=\delta_{j l}
$$

Consequently

$$
u_{i}^{(m)}(z, \rho)=f_{m}(z, \rho) \sum_{k=0}^{n-1} \beta_{j k}(\rho)\left\{\left[\sum_{l=0}^{n-1} \hat{c}_{k l} y_{l}{ }^{(m)}\right]+\frac{E(z, \rho)}{\rho^{n / p}}\right\} .
$$

Now let $z=0$; then $t=0, y_{l}^{(m)}=\delta_{l m}$, and thus

$$
\delta_{j m}=f_{m}(0, \rho) \sum_{k=0}^{n-1} \beta_{j k}(\rho)\left\{\hat{\imath}_{k m}+\frac{E(\rho)}{\rho^{n / p}}\right\} .
$$

This may be written

$$
\delta_{j m}=\sum_{k=0}^{n-1}\left\{f_{j}(0, \rho) \beta_{j k}(\rho)\right\}\left\{\hat{c}_{k m}+\frac{E(\rho)}{\rho^{n / p}}\right\} .
$$

Compare with this the system

$$
\delta_{j m}=\sum_{k=0}^{n-1} \chi_{j k} \hat{c}_{k m} \quad(m=0,1, \cdots n-1),
$$

whose solutions are evidently

$$
\chi_{j k}=c_{j k} .
$$

It follows that a system

$$
\delta_{j m}=\sum_{k=0}^{n-1} \chi_{j k}(\rho)\left\{\hat{c}_{k m}+\frac{E(\rho)}{\rho^{n / p}}\right\}
$$

has solutions

and hence

$$
\chi_{j k}=c_{j k}+\frac{E(\rho)}{\rho^{n / p}}
$$

$$
\beta_{j k}(\rho)=\frac{1}{f_{j}(0, \rho)}\left\{c_{j k}+\frac{E(\rho)}{\rho^{n / p}}\right\} .
$$

Substitution of this value in (35) and interchange of the order of summation yields the equation

or

$$
u_{j}^{(m)}(z, \rho)=\frac{f_{m}(z, \rho)}{f_{j}(0, \rho)}\left\{\left[\sum_{l=0}^{n-1} y_{l}^{(m)} \sum_{k=0}^{n-1} c_{j k} \hat{c}_{k l}\right]+\frac{E(z, \rho)}{\rho^{n / p}}\right\}
$$




$$
u_{j}^{(m)}(z, \rho)=\frac{f_{m}(z, \rho)}{f_{j}(0, \rho)}\left\{y_{j}^{(m)}(t)+\frac{E(z, \rho)}{\rho^{n / p}}\right\} .
$$

If we now resume the subscripts $Z, \tau$, we find that in the last equation they apply only to the $E$-function. But we were careful to note that the $E$-functions of Theorem 1 were uniformly bounded for all $Z, \tau$. Consequently the present $E$-function inherits the same property, that is, it is bounded uniformly for all $(z, \rho)$ for which $z$ is in $R_{z},|\rho|>\rho_{0}, \lambda$ is an admitted value, and $|\xi|<N$. On this result we base

THEOREM 2. For admitted values of $\arg \rho,|\rho|>\rho_{0}$, and $z$ in $R_{z}$, the solutions of the given equation principal at the origin, $\left\{u_{j}^{(m)}(0, \rho)=\delta_{j m}, j, m=0,1, \cdots\right.$, $n-1\}$, have the form

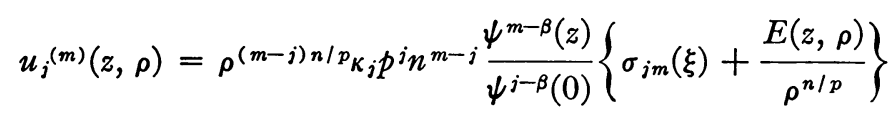

when $|\xi|<N$. The series $\sigma_{j m}(\xi)$ and constants $\kappa_{j}$ are defined by equations (5) et seq.

In unabbreviated notation, equation.(36) becomes

$$
{ }_{z, \tau} \beta_{j k}(\rho)=\frac{\rho^{-j n / p}\left(\frac{p}{n}\right)^{j \nu / p}}{\psi^{j-\beta}(0)}\left\{{ }_{r} c_{j k}+\frac{E(\rho)}{\rho^{n / p}}\right\},
$$

where the $E$-function is uniformly bounded for all $Z, \tau$. Taking this and the forms (33) into (34), we obtain

$$
u_{j}{ }^{(m)}(z, \rho)=\rho^{(m-j) n / p}\left(\frac{p}{n}\right)^{j \nu / p} \frac{\psi^{m-\beta}(z)}{\psi^{j-\beta}(0)} \frac{{ }_{r} H_{j m}(z, \rho)}{\xi^{(\beta-m) / p}},
$$

where

$$
{ }_{r} H_{j m}(z, \rho) \equiv \sum_{k=0}^{n-1} \omega_{k}{ }^{m} e^{\omega_{k} \xi}\left\{{ }_{r} c_{j k}+\frac{E(\xi)}{\xi}+\frac{E(z, \rho)}{\rho^{n / p}}\right\},
$$

and, for admitted $\lambda$ and $|\rho|>\rho_{0}$, the $E$-functions are bounded uniformly when $\xi$ is in $S_{\tau}$ and $|\xi| \geqq N$.

We recall that a region $T_{q}$ is bisected by the ray $L_{2 q}$ (see $\S 3$ ), but that in both halves the number $\omega_{0}$ of the dominant solution has the same value $\omega^{q}$ (see (10)). In $T_{q}$

$$
R\left(\omega_{k} \xi-\omega_{0} \xi\right) \leqq h|\xi|, \quad h<0, \quad k \neq 0 ;
$$

also, the multiplier of the dominant solution is the same in both halves of $T_{q}$, 
namely, ${ }_{q} \gamma_{j} \neq 0$; hence with $\tau=2 q$ or $2 q+1$, depending on which half of $T_{q}$ contains $\xi$,

$$
{ }_{{ }_{\tau}} H_{j m}(z, \rho)={ }_{a} \gamma_{j} \omega^{q m} e^{\omega^{\alpha} \xi}\left\{1+\frac{E(\xi)}{\xi}+\frac{E(z, \rho)}{\rho^{n / p}}\right\} .
$$

By a similar method we can treat the case when $\xi$ is in the sector $D_{q, q+1}$ between $T_{q}$ and $T_{q+1}$; the results are contained in

TheOREM 3. When $|\xi| \geqq N$, and for admitted values of arg $\rho,|\rho|>\rho_{0}$, and $z$ in $R_{z}$, the principal solutions of the given equation are of the form

$$
u_{j}^{(m)}(z, \rho)={ }_{q} \Psi_{j m}(z, \rho)\left\{1+\frac{E(\xi)}{\xi}+\frac{E(z, \rho)}{\rho^{n / p}}\right\}
$$

for $\xi$ in $T_{q}$, and

$$
\begin{aligned}
u_{j}{ }^{(m)}(z, \rho)= & { }_{q} \Psi_{j m}(z, \rho)\left\{1+\frac{E_{1}(\xi)}{\xi}+\frac{E_{1}(z, \rho)}{\rho^{n / p}}\right\} \\
& +{ }_{q+1} \Psi_{j m}(z, \rho)\left\{1+\frac{E_{2}(\xi)}{\xi}+\frac{E_{2}(z, \rho)}{\rho^{n / p}}\right\}
\end{aligned}
$$

for $\xi$ in $D_{q, q+1}$, where

$$
{ }_{q} \Psi_{j m}(z, \rho) \equiv \frac{\rho^{(m-j) n / p} \kappa_{j} p^{j} e^{(2 \pi i q / n)[(n j+\nu \beta) / p-m]}}{n^{1 / 2+\nu(j-\beta) / p}(2 \pi)^{\beta}} \cdot \frac{\psi^{m-\beta}(z)}{\psi^{j-\beta}(0)} \cdot \frac{e^{\omega q \xi}}{\xi^{\nu(\beta-m) / p}} .
$$

12. Discussion of results. Two sectors $T_{q}$ and $T_{q+n}$ lie in the same position but in adjacent sheets of the $\xi$-surface. The analytic expressions given by Theorem 3 for $u_{j}^{(m)}(z, \rho)$ differ for the two regions by a factor $e^{2 \pi i \alpha_{j}}$, where

$$
\alpha_{j} \equiv \frac{n j+\nu \beta}{p}=\frac{2 n j+\nu(n-1)}{2(n+\nu)} ;
$$

that is, the expression for $\xi$ in $T_{q+n}$ is $e^{2 \pi i \alpha_{i}}$ times that for $\xi$ in $T_{q}$. This factor may be regarded as a measure of the Stokes phenomenon.

We see that $\alpha_{j}$ is independent of the region $T_{q}$ as well as the order $m$ of the derivative. Evidently $e^{2 \pi i \alpha_{j}}$ will be unity, in other words, there will be no Stokes phenomenon, if and only if $\alpha_{j}$ is integral. Thus, if $\nu=0$, none of the solutions will show a Stokes phenomenon. However, if $\nu>0$, not all the solutions can lack it, since $\alpha_{j}$ and $\alpha_{j+1}$ differ by $n / p$ and hence the $\alpha_{j}$ 's cannot all be integers. In particular, for $n=2$ every principal solution manifests the 
phenomenon, but for any $n>2$ there are always some $(\nu, j)$ for which it fails to appear.*

The results of Theorem 3 could have been expressed in the form $\dagger$

$$
\begin{aligned}
u_{j}^{(m)}(z, \rho)= & \rho^{(m-j) n / p}\left(\frac{p}{n}\right)^{j \nu / p} \frac{\psi^{m-\beta}(z)}{\psi^{j-\beta}(0)} \\
& \cdot \frac{1}{\xi^{(\beta-m) / p}} \sum_{l=0}^{n-1}{ }_{\tau} d_{j l} \omega^{l m} e^{\omega l \xi}\left\{1+\frac{E(\xi)}{\xi}+\frac{E(z, \rho)}{\rho^{n / p}}\right\}
\end{aligned}
$$

for $|\xi| \geqq N$, where the multiplier ${ }_{\tau} d_{j l}$ depends on which region $S_{\tau}$ contains $\xi$. The set $\left\{{ }_{r} d_{j l}\right\}$ is a permutation of the set $\left\{{ }_{r} c_{j l}\right\}$, such that, if $\omega_{r}=\omega^{l}$ in $S_{r}$, then ${ }_{r} c_{j r}={ }_{\tau} d_{j l}$. To see that this is entirely equivalent to Theorem 3 , we need only note that if $\xi$ is in $T_{q}$, the sum on the right of (41) may be replaced by the dominant term, the other terms being absorbed into the corresponding $E(z, \rho)$, giving formula (38). If $\xi$ is in a $D$-sector, two terms must be kept as in formula (39).

Consider one of the terms of (41) for fixed $l$. It is dominant in the regions $T_{q_{l}}$, where $q_{l} \equiv l(\bmod n)$. The sectors $T_{q_{l}}$ are all in the same position but in different sheets of the $\xi$-surface, and are bisected by the rays $L_{2 q_{l}}$. The rays $L_{\tau_{l}}, \tau_{l} \equiv 2 l-n(\bmod 2 n)$, leave the origin in the opposite direction. With reference to the equation (41), the Stokes phenomenon could evidently be described as follows: The value of the multiplier ${ }_{r} d_{j l}$ need be changed only when crossing a ray $L_{\tau_{l}}$, and then by the factor $e^{2 \pi i \alpha_{j}}$. Instead of this particular ray, it would be equally compatible with our results to employ as Stokes line any simple curve from 0 to $\infty$ on the $\xi$-surface not lying in $T_{q_{l}}$ and the adjacent $D$-sectors. However, this location of the Stokes lines is more general than would be warranted if it were not for a certain insufficiency in our results, which we now bring to attention.

Let $u(z, \rho)$ be the solution of the given equation satisfying the boundary conditions

Then

$$
u^{(m)}(0, \rho)=h_{m}(\rho) \quad(m=0,1, \cdots, n-1) .
$$

$$
u^{(m)}(z, \rho)=\sum_{j=0}^{n-1} h_{j}(\rho) u_{j}^{(m)}(z, \rho) .
$$

* If $n=2, \nu>0$, then $0<\alpha_{i}<1, j=0,1$.

If $n>2, \nu>0$, we have the following:

Case 1: $n$ odd. For every $\nu$ at least one $\alpha_{j}$ is integral, namely, for $j=(n-1) / 2$.

Case 2: $n$ even, $\nu$ odd. No $\alpha_{j}$ can be integral, since $\alpha_{j}=$ odd/even.

Case 3: $n$ even, $\nu$ even. There may or may not exist integral $\alpha_{j}$ for a definite $\nu$. However, for $\nu=n(n-2), \alpha_{0}$ and $\alpha_{n-1}$ are integral.

$\dagger$ It is understood that the discussion in this section applies only to such $\rho$ as have admitted $\lambda \equiv \arg \rho$ and $|\rho|>\rho_{0}$. 
Using the forms of Theorem 3, we have for $|\xi| \geqq N$ and $\xi$ in $T_{q}$,

$$
u^{(m)}(z, \rho)={ }_{q} A_{m}(z, \rho) \sum_{j=0}^{n-1} h_{j}(\rho){ }_{Q} B_{j}(\rho)\left\{1+\frac{E_{j}(\xi)}{\xi}+\frac{E_{j}(z, \rho)}{\rho^{n / p}}\right\},
$$

where

$$
{ }_{q} B_{j}(\rho) \equiv \rho^{-j n / p} \frac{\kappa_{j} p^{j} e^{2 \pi i q j / p}}{n^{v i / p} \psi^{j}(0)} .
$$

In order that the expression (42) be explicit to a factor

$$
\left\{1+\frac{E(\xi)}{\xi}+\frac{E(z, \rho)}{\rho^{n / p}}\right\}
$$

the following condition on the boundary values $\left\{h_{j}(\rho)\right\}$ is sufficient, and, in the absence of further knowledge of the $E_{j}$-functions, also necessary: The quantities

$$
{ }_{q} C_{j}(\rho) \equiv \frac{\rho^{-j n / p} h_{j}(\rho)}{\sum_{l=0}^{n-1}{ }_{q} B_{l}(\rho) h_{l}(\rho)} \quad(j=0,1, \cdots, n-1)
$$

are bounded. This follows from noting that the expression (42) is explicit to a factor

$$
1+\sum_{j=0}^{n-1}{ }_{2} C_{j}(\rho)\left\{\frac{\bar{E}_{j}(\xi)}{\xi}+\frac{\bar{E}_{j}(z, \rho)}{\rho^{n / p}}\right\}
$$

where the $\bar{E}$ 's are constant multiples of the $E$ 's. For such $\rho$ as satisfy the conditions, and $|\xi| \geqq N, u^{(m)}(z, \rho)$ then has the form

$$
u^{(m)}(z, \rho)=\left\{{ }_{q} A_{m}(z, \rho) \sum_{j=0}^{n-1} h_{j}(\rho){ }_{q} B_{j}(\rho)\right\}\left\{1+\frac{E(\xi)}{\xi}+\frac{E(z, \rho)}{\rho^{n / p}}\right\}
$$

when $\xi$ is in $T_{q}$, while for $\xi$ in $D_{\bar{q}, \bar{q}+1}$ the form is* the sum of two such expressions written respectively for $q=\bar{q}$ and $\bar{q}+1$. From this it is easily seen that our formulas are not explicit to a factor (43) in a region which is not zero-free for $u^{(m)}(z, \rho), m=0,1, \cdots, n-1$.

This insufficiency of our formulas to describe the most general solution bears on the freedom of locating the Stokes lines: The only time we would need to know ${ }_{\tau} d_{j l}$ outside a region $T_{q_{l}}$ and the adjoining $D$-sectors would be when we have such a combination of the principal solutions,

$$
u^{(m)}(z, \rho)=\sum_{j=0}^{n-1} h_{j}(\rho) u_{j}^{(m)}(z, \rho)=\sum_{k=0}^{n-1} z_{, \tau} \beta_{k}(\rho)_{z, \tau} \tilde{u}_{k}^{(m)}(z, \rho)
$$

\footnotetext{
* Providing ${ }_{q} C_{j}(\rho)$ is bounded for $q=\bar{q}$ and $\bar{q}+1$.
} 
that the dominant solution ${ }_{z, \tau} \tilde{u}_{0}^{(m)}(z, \rho)$ fails to dominate the expression. But this can happen only in a case not covered by our formulas because of a lack of knowledge about the $E$-functions. If it were not for this lack, the location of the Stokes lines would have to be more restricted; thus, for the $t$-equation it can be shown that the Stokes lines for ${ }_{\tau} d_{j l}$ must be taken to lie in those regions of the $\xi$-surface which are reflections of $T_{q_{l}}$ in the origin.

We shall conclude with some mention of how our results specialize. Consider the case when $\nu=0$. We have already seen that there is then no Stokes phenomenon. A great simplification of the results can now be made since it is possible to assume $|\xi| \geqq N$ always, by taking $N=0$. The constant $N$ was introduced first in $\$ 3$, where it can be taken as zero, since the series

$$
s_{k}^{(m)}(t)=\omega_{k}^{m} e^{\omega_{k} \xi}\left\{1+\frac{0}{\xi}+\frac{0}{\xi^{2}}+\cdots\right\}
$$

are now true solutions, ${ }^{*}$

$$
{ }_{\tau} \tilde{y}_{k}^{(m)}(t)=s_{k}^{(m)}(t) .
$$

We again encounter it in $\S 6$, where we find formula (19) with $E(\xi) \equiv 0$ is valid for all $\xi$. The comparison in Chapter IV can now be carried through with $N=0$, and again the formula (33) of Theorem 1 works for all $\xi$ with $E(\xi) \equiv 0$. The method of deriving the forms of the multipliers ${ }_{z, r} \beta_{j k}(\rho)$ in $\$ 11$ is then applicable without the assumption $|\xi|<N$. We can now dispense with Theorem 2 and use Theorem 3 for all $\xi$ with $N=0$, noting that

$$
E(\xi) \equiv E_{1}(\xi) \equiv E_{2}(\xi) \equiv 0,
$$

and that the formula (40) now assumes the simple form

$$
{ }_{q} \Psi_{j m}(z, \rho)=\rho^{m-j} \frac{e^{2 \pi i q(j-m) / n}}{n} \cdot \frac{\phi^{m-\beta}(z)}{\phi^{j-\beta}(0)} e^{\omega^{\alpha} \xi} .
$$

Finally, a word about the case when the coefficient $\phi^{n}(z)$ is real on the real axis, or real except for a constant imaginary factor. By the transfer of a constant factor to the parameter $\rho^{n}, \phi_{1}{ }^{n}(z)$ may be made real and positive on the real axis. The cut in the $z$-plane can now be taken along the negative real axis and need not be deformed. The positive and negative real axis map respectively into the rays arg $\Phi=0, p / n \pi$. It follows that if one is interested only in real $z$, all values (12) of $\arg \rho$ may be admitted.

* Note $\xi \equiv t$; also $p=n$ and $\psi(z) \equiv \phi(z)$.

UNIVERSITY OF WISCONSIN, Madison, Wis. 